\title{
All-trans retinoic acid enhances temozolomide-induced autophagy in human glioma cells U251 via targeting Keap1/Nrf2/ARE signaling pathway
}

\author{
LIN SHI $^{1 *}$, HONGYUAN LI ${ }^{1 *}$ and YANG ZHAN ${ }^{2}$ \\ Departments of ${ }^{1}$ Neurosurgery and ${ }^{2}$ Pathology, Dezhou People's Hospital, Dezhou, Shandong 253014, P.R. China
}

Received January 18, 2017; Accepted May 17, 2017

DOI: $10.3892 / \mathrm{ol} .2017 .6482$

\begin{abstract}
The present study evaluated the retinoic acid (RA) enhancement of temozolomide (TMZ) effects on the human glioma cells U251 and explored its underlying molecular mechanism. The cell growth was detected using the MTT assay and the cell cycle was assessed by flow cytometry. Cell apoptosis was analyzed by Annexin V/propidium iodide staining, and the cell morphology was evaluated using transmission electron microscopy (TEM). Additionally, reverse transcription-PCR and western blot analysis were applied to detect the mRNA and protein levels. The RA treatment in combination with TMZ in the human U251 cells further inhibited cells growth, arresting cell cycle progression at the G0/G1 phase, and significantly induced apoptosis of U251 cells. After the RA+TMZ treatment of U251 cells, autophagy associated proteins Beclin 1 and LC3B were significantly increased, and the TEM analysis were consistent with autophagy protein levels. Moreover, Keap1/Nrf2/ARE expression was downregulated significantly, indicating the involvement in the mechanisms that RA treatment could enhance the TMZ effects on U251 cells. RA treatment in combination with TMZ may provide some experimental evidence for the possible effect of RA+TMZ against the growth and the proliferation of glioma cells. Therefore, the RA+TMZ administration may have potential utility for glioblastoma treatment.
\end{abstract}

\section{Introduction}

Glioblastoma (GBM) is an aggressive astrocytic neoplasm and one of the main contributors to cancer-associated deaths in

Correspondence to: Dr Yang Zhan, Department of Pathology, Dezhou People's Hospital, 1751 Xinhu Street, Dezhou, Shandong 253014, P.R. China

E-mail: huan25804040xi@163.com

*Contributed equally

Key words: human glioblastoma, retinoic acid, temozolomide, autophagy, Keap1/Nrf2/ARE adult and pediatric populations (1). Median survival time of GBM patients is approximately 12-15 months after the first diagnosis (2). Conventional treatments for GBM patients are mostly surgical tumor excision followed by chemotherapy or radiation therapy (3). Although the therapies have increased the survival time to approximately 14.6 months, the advantages are unconspicuous (4). Temozolomide (TMZ) was approved for use in radiotherapy for treatment of newly diagnosed GBM in adults and as a maintenance therapy in 2005 (5). TMZ is the most commonly used and first line chemotherapeutic agent for GBM patients, though some other chemotherapeutic drugs could be found as pharmaceutical options $(6,7)$.

Retinoic acid (RA) is a chemotherapeutic and chemopreventive agent (8). It can inhibit tumorigenesis via suppressing the cell growth and stimulating cell differentiation (9). Besides, RA also promotes cellular apoptosis which may be a reason of its antitumor characteristics (10). RA mediated signaling influences the development of the central nervous system through regulating neuronal differentiation process (11), and at early stages adjusting the neurogenesis of adults (12). Autophagy is usually activated in tumors by radiation or chemotherapy $(13,14)$, and this process acts as a potential protector for cancer treatment. Autophagy has been thought as a pro-death or pro-survival response to several external stresses, such as the radiotherapy and chemotherapy at both organic and cellular levels (15). The underlying mechanisms that autophagy has such as seemingly opposite functions remained elusive. A study reported that autophagy relative pathways operate to supply cells with metabolic substrates, leading to cell maintenance in many moderate stimulus conditions (16). However, various studies reported that autophagy, if uncontrolled, also became a contributor to cell death that could occur in the absence of detectable apoptosis signs (17). In addition, one important autophagy hallmark is the convertion of light chain 3A to its lapidated form LC3B (18). Besides, LC3B is the first identified mammalian protein that related to autophagy (19). Beclin 1 is another important autophagyassociated protein, which has been reported to play a central role in neurodegeneration prevention (20).

In the present study, we investigated the the RA potential therapeutic function to enhance the TMZ effects on the U251 cells, and explored the growth, proliferation, and apoptosis of the U251 cells after the RA+TMZ treatment. Moreover, the role of the RA+TMZ was also elucidated on the regulation 
of autophagy associated proteins Beclin 1 and LC3B. Most importantly, the underlying mechanisms of RA enhancement to TMZ effects on U251 cells were investigated.

\section{Materials and methods}

Cell culture. The human U251 glioma cells line was purchased from the American Type Culture Collection (Rockville, MD, USA). The cancer cell line was cultured in RPMI-1640 containing $10 \%$ fetal bovine serum (FBS), $50 \mu \mathrm{g} / \mathrm{ml}$ amphotericin $\mathrm{B}$ and $100 \mathrm{U} / \mathrm{ml}$ penicillin, and maintained at $37^{\circ} \mathrm{C}$ in $5 \% \mathrm{CO}_{2}$ incubator. The control cells were untreated in medium containing vehicle dimethyl sulfoxide (DMSO).

MTT assay. Cells were plated in 24-well plates $\left(5 \times 10^{3}\right.$ cells per well). TMZ and RA at various concentrations were added after cells attachment to the plates. Then after overnight culture, baseline values were obtained by an MTT assay (Life Technologies, Grand Island, NY, USA). It is a colorimetric assay to test viable cells by measuring formazan reduced from MTT. After $20 \mathrm{~min}$ of incubation with MTT, we extracted formazan by DMSO and tested the optical density at $540 \mathrm{~nm}$. MTT assay was performed at $48 \mathrm{~h}$. Experiments were performed in triplicate.

Cell cycle analysis by flow cytometry. U251 cells were collected following the TMZ $(400 \mu \mathrm{M})$ and RA $(10 \mu \mathrm{M})$ and incubated $48 \mathrm{~h}$. Then the cells were fixed in the ice-cold ethanol $(70 \%)$ at $-20^{\circ} \mathrm{C}$ overnight. The next morning, the cells were first incubated in the $10 \mu \mathrm{M}$ RNAse at $37^{\circ} \mathrm{C}$ for $30 \mathrm{~min}$, then stained with propidium iodide at $4^{\circ} \mathrm{C}$ for $1 \mathrm{~h}$. Cell cycle analysis was carried out on a FACSCalibur flow cytometer (BD Biosciences, San Diego, CA, USA) and the obtained data were analyzed using CellQuest ${ }^{\mathrm{TM}}$ software (BD Biosciences).

Cell apoptosis by Annexin V/propidium iodide (PI) double staining. Following treatment with $\mathrm{TMZ}(400 \mu \mathrm{M})$ and RA $(10 \mu \mathrm{M})$ and incubation for $48 \mathrm{~h}$, the cells for apoptosis analysis were first resuspended in Annexin V binding buffer, and then stained with the FITC-conjugated Annexin V and PI simultaneously at $37^{\circ} \mathrm{C}$ for $15 \mathrm{~min}$ in the dark before adding the binding buffer. Then the apoptotic cells were identified using the flow cytometry analysis on a FACSCalibur system (FacsScan; BD Biosciences).

Transmission electron microscopy (TEM). For the TEM analysis of cell morphological features, cells were first fixed in 3\% glutaraldehyde followed by $1 \%$ osmium tetroxide, then dehydrated in the graded alcohols and embedded in Epon (Agar Scientific, Stansted, UK). The sections were stained in lead citrate and uranyl acetate and were finally observed with the Morgagni 268d transmission electron microscope (FEI, Hillsboro, OR, USA).

Drug treatment. DMSO, potassium phosphates, Propidium iodide, Tris- $\mathrm{HCl}$, ribonuclease-A, Triton X-100 and Trypan blue were obtained from Sigma Chemical Co. (St. Louis, MO, USA). L-glutamine, DMEM, FBS and penicillin-streptomycin were purchased from Gibco Invitrogen Corp. (Grand Island, NY, USA). TMZ and RA were obtained from Sigma Chemical Co. and dissolved in DMSO to the optimal working concentration.
Western blot analysis. After treatment, the cells were collected and the protein extracted with RIPA lysis buffer. The protein concentrations were quantified by the BCA method. Protein samples $(30 \mu \mathrm{g})$ were run on $10 \%$ gels, and then transferred to the PVDF membrane. After $1 \mathrm{~h}$ of blocking with the 5\% non-fat milk, the membranes were incubated with the primary rabbit anti-LC3B, the rabbit anti-Beclin 1 antibody, the rabbit anti-Keap1 antibody, the rabbit anti-Nrf2 antibody, the rabbit anti-ARE antibody (1:1,000; Abcam, Cambridge, MA, USA) at $4^{\circ} \mathrm{C}$ overnight. Washing in TBST three times, the membranes were then incubated with a peroxidase labeled secondary antibody (1:5,000; Santa Cruz Biotechnology, Inc., Dallas, TX, USA) for $2 \mathrm{~h}$. The bands were washed again, enhanced with chemiluminescence reagents and visualized with the ChemiDoc $^{\mathrm{TM}}$ MP Imaging System (Bio-Rad, Berkeley, CA, USA).

Reverse-transcription PCR. After treatment, the cortex was collected to determine the expression of Keap1/Nrf2/ARE mRNA by RT-PCR. Total RNA was extracted by the TRIzol RNA extraction reagent (Invitrogen, Carlsbad, CA, USA) following the protocols and quantified by spectrophotometer method. Purified RNA with equal volume was reverse transcribed (RevertAid Fist Strand cDNA Synthesis kit, K1622; Thermo Fisher Scientific, Waltham, MA, USA). RT-PCR analysis was performed using a PCR Thermal Cycler Dice instrument (Takara Bio, Inc., Otsu, Japan).

Statistical analysis. All data were expressed as the mean \pm standard error of the mean (SEM), and were analyzed using a one-way analysis of variance followed by Bonferroni-Dunn correction. Statistical analysis was performed using SPSS software, version 20.0 (SPSS, Inc., Chicago, IL, USA).

\section{Results}

RA enhances the TMZ effects on inhibition of the U251 cell proliferation. To verify if RA has additive, synergistic or antagonistic effects after administration with TMZ, U251 cells were treated with RA and TMZ at various concentrations and incubated for $48 \mathrm{~h}$. As shown in Fig. 1A, the TMZ IC50 was $691.6 \mu \mathrm{M}$ while the TMZ+RA IC50 was TMZ $386.7 \mu \mathrm{M}$ and RA $9.6 \mu \mathrm{M}$ after incubation for $48 \mathrm{~h}$. As Fig. 2B shown, the MTT assay further conformed that RA enhanced the TMZ effects on inhibition of the U251 cells proliferation.

$R A$ enhances the TMZ effects on modulation of the U25I cell cycle. The U251 cells were treated with RA and TMZ at an appropriate ratio according to their IC50, respectively, and incubated for $48 \mathrm{~h}$. Then the flow cytometry analysis was performed to investigate the U251 cell cycle according to the cell cycle stage distribution (G0, G1, S, G2 and M). We found a slight G0/G1 phase arrest in the TMZ group and a marked arrest in the RA+TMZ group, elucidating that RA could enhance the TMZ effects on modulation of the U251 cell cycle (Fig. 2).

$R A$ enhances the TMZ effects on induction of U251 cell apoptosis. With the Annexin V/FITC and PI method, the RA ability to enhance the TMZ effects on inducing U251 cell apoptosis 
A

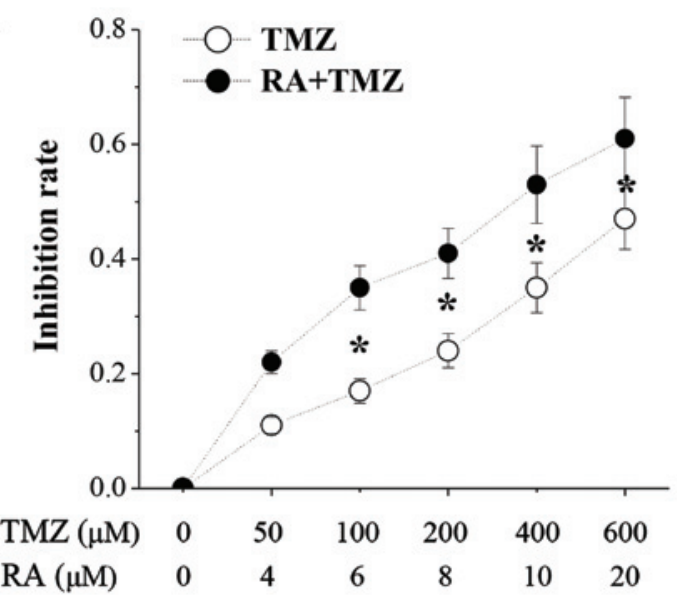

B
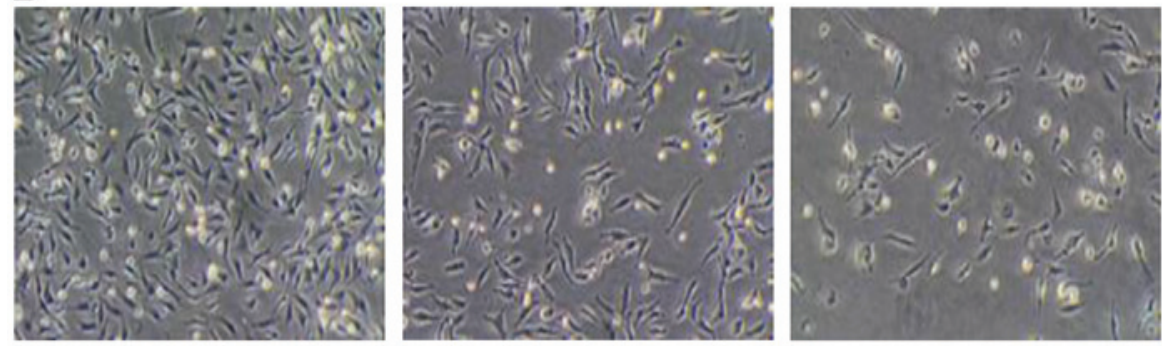

Figure 1. RA enhances the effects of TMZ on the U251 cell proliferation. (A) Growth inhibition of the U251 cells was analysed following exposure to TMZ treatment and RA+TMZ administration for $48 \mathrm{~h}$. (B) Morphological changes of cells following exposure to TMZ treatment and RA+TMZ administration for 48 h. Statistically significant difference ( $(\mathrm{P}<0.05)$. RA, retinoic acid; TMZ, temozolomide.
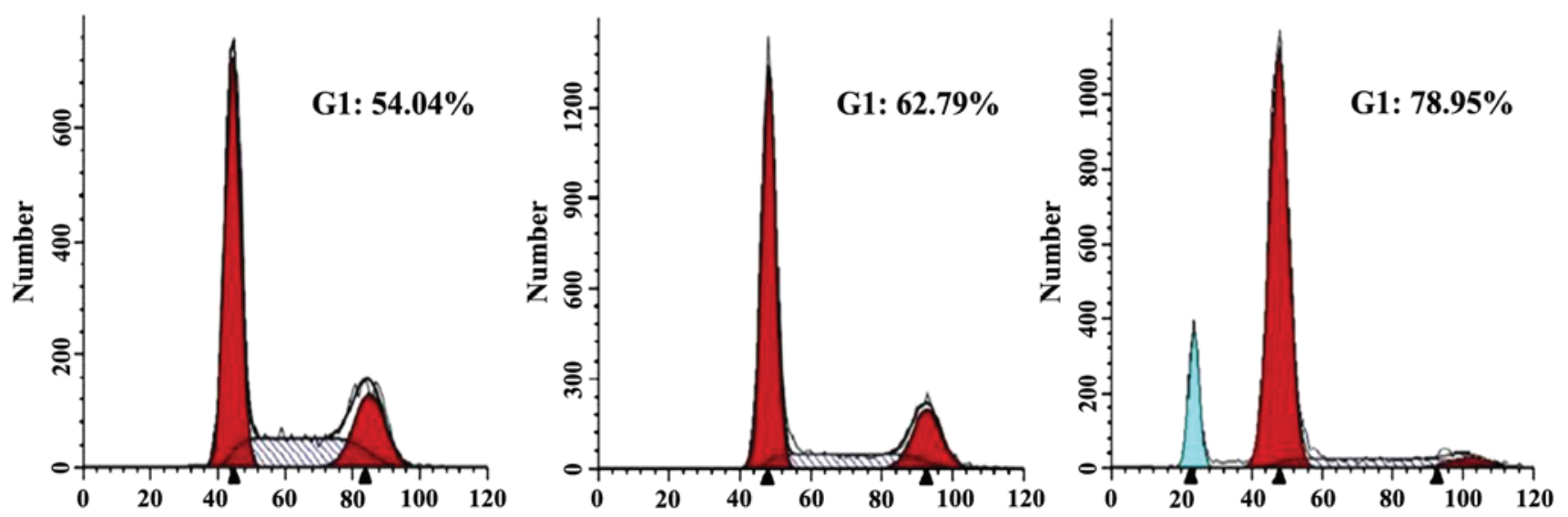

Figure 2. RA enhances the effects of TMZ on the U251 cell cycle. Flow cytometry analysis on cell cycle progression in U251 cells was carried out after incubation with TMZ or RA+TMZ for $48 \mathrm{~h}$. Representative bar chart demonstrated significant cell cycle arrest at the G1 phase. RA, retinoic acid; TMZ, temozolomide.

was explored. U251 cell apoptosis was slightly induced in the TMZ group while significantly induced by the RA+TMZ treatment (Fig. 3A). As shown in Fig. 3B, the number of apoptotic cells was markedly increased after treatment with $10 \mu \mathrm{M}$ RA $+400 \mu \mathrm{M}$ TMZ compared with the TMZ group $(21.6 \pm 1.5 \%$ vs. $12.4 \pm 1.1 \%)$.

RA enhances the TMZ effects on induction of U251 cell autophagy. In order to assess the presumed autophagy mechanism in U251 cells, we detected the protein leves of autophagy associated proteins Beclin 1 and LC3B with western blot analysis. The LC3B and Beclin 1 levels were slightly upregualated in the TMZ group while significantly increased by the RA+TMZ treatment (Fig. 4A). Furthermore, the results of ultrastructural TEM analysis were consistent with autophagy induction, showing an extensive cytoplasm vacuolization and many single-membrane autophagolysosomes containing cellular debris (Fig. 4B).

RA enhances the effects of TMZ reducing the Keap1/Nrf2/ARE signaling. Western blot analysis and RT-PCR were applied to assess the mRNA and protein levels of Keap1/Nrf2/ARE to 

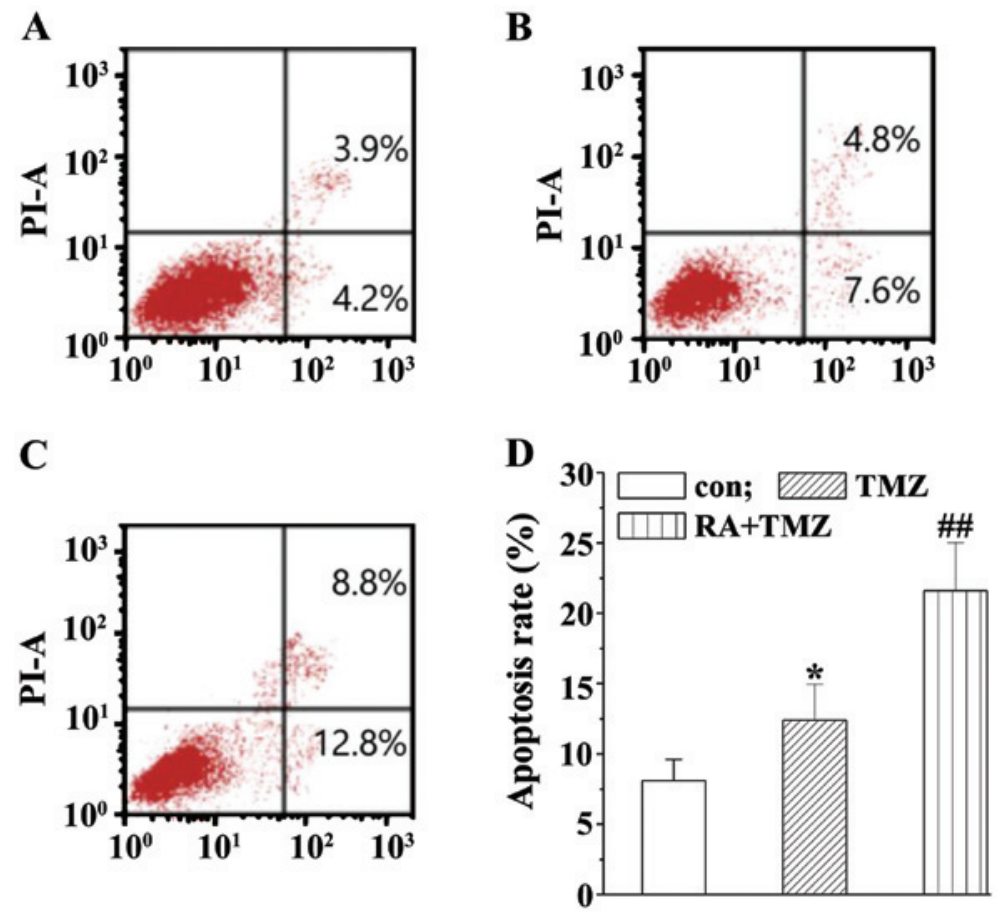

Figure 3. RA enhances the effects of TMZ on U251 cells apoptosis. Flow cytometric analysis of apoptosis in the (A) control group, (B) TMZ group and (C) RA+TMZ group. (D) Percentage of apoptotic cells after different treatment. Statistically significant difference (" $P<0.05)$ between the TMZ group and the control group. Statistically significant difference $\left({ }^{\# \#} \mathrm{P}<0.01\right)$ between TMZ group and RA+TMZ group. RA, retinoic acid; TMZ, temozolomide.

A
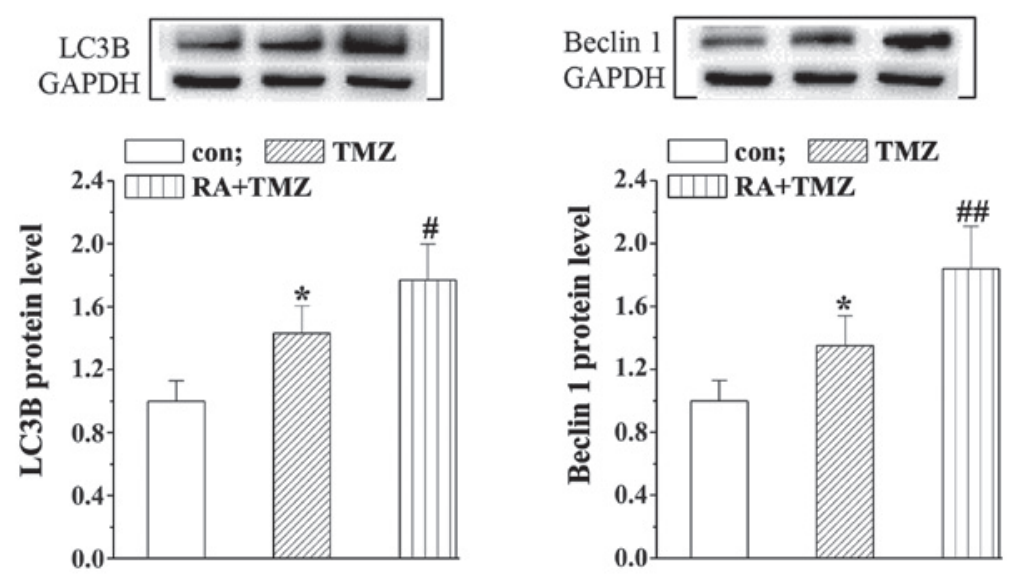

B
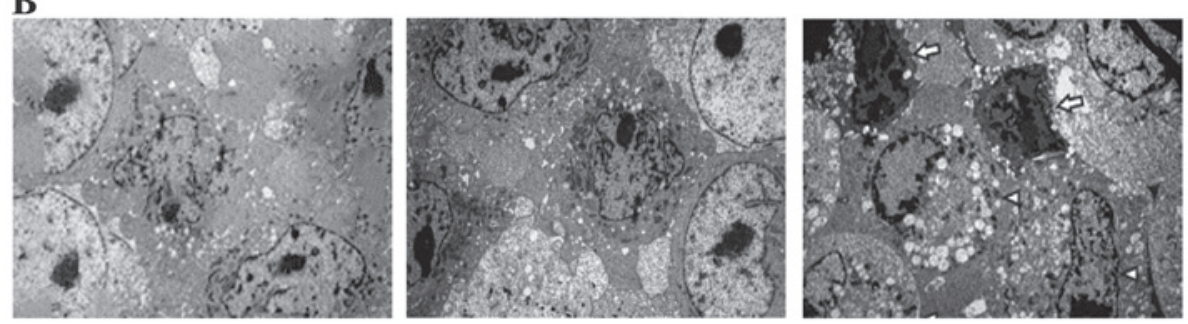

Figure 4. RA enhances the effects of TMZ on U251 cell autophagy. (A) Autophagy-associated proteins LC3B and Beclin 1 protein levels were detected using western blot analysis in the treatment and control groups. (B) Ultrastructural TEM analysis was performed in the treatment and control groups. Statistically significant difference $\left({ }^{*} \mathrm{P}<0.05\right)$ between the TMZ group and the control group. Statistically significant difference $\left({ }^{\#} \mathrm{P}<0.05\right.$, $\left.{ }^{\# \#} \mathrm{P}<0.01\right)$ between $\mathrm{TMZ}$ group and RA+TMZ group. RA, retinoic acid; TMZ, temozolomide.

explore the underlying mechanisms of RA in combination with TMZ. As shown in Fig. 5, the Keap1/Nrf2/ARE expression was slightly downregulated in the TMZ group while markedly reduced by the RA+TMZ treatment.

\section{Discussion}

In the present study, the RA potential therapeutic function to enhance the TMZ effects on the U251 cells were evaluated. 
A
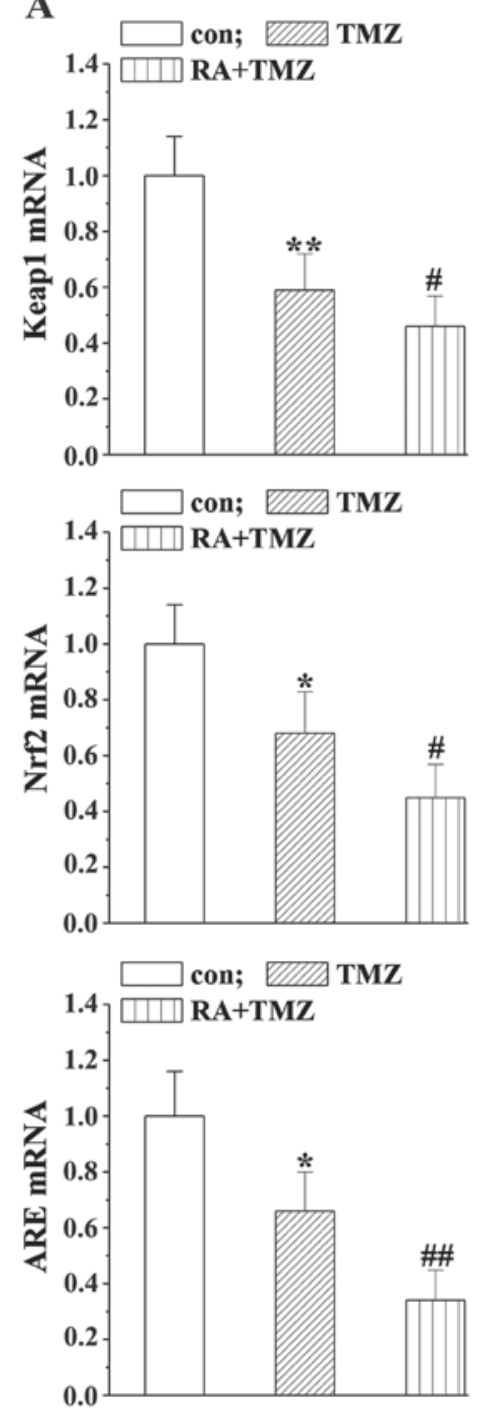
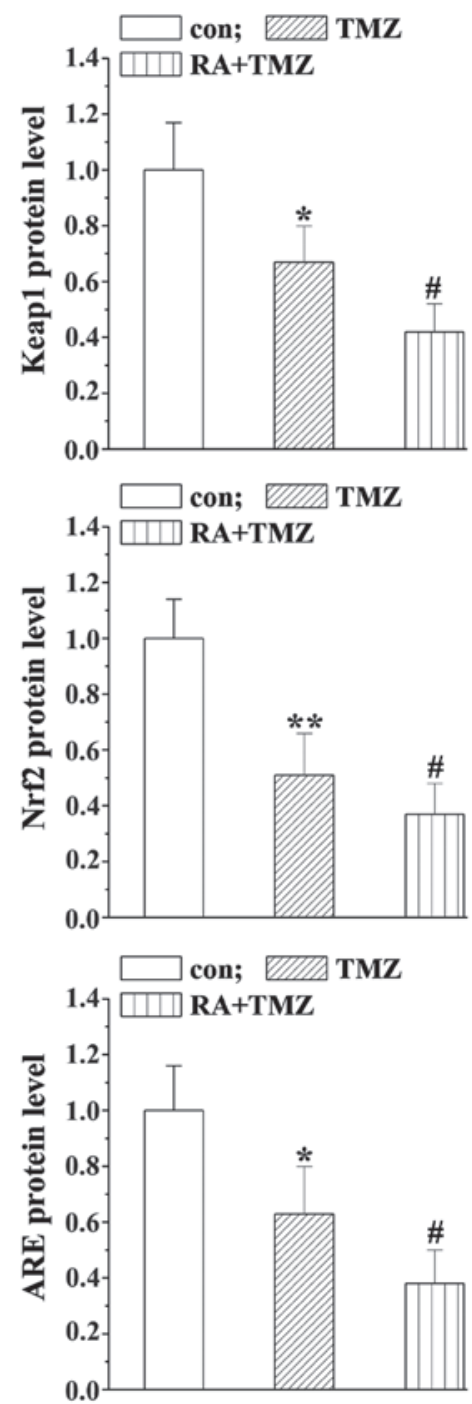

B

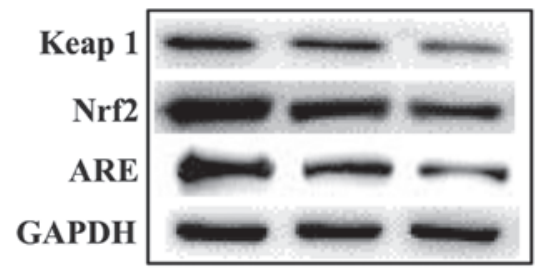

Figure 5. RA enhances the effects of TMZ on Keap1/Nrf2/ARE signaling. (A) Keap1/Nrf2/ARE mRNA levels detected by RT-PCR. (B) Protein levels were detected using western blot analysis. Bar charts show relative protein levels in the treatment and control groups. Statistically significant difference ("P $<0.05$, $\left.{ }^{* *} \mathrm{P}<0.01\right)$ between the TMZ group and the control group. Statistically significant difference $\left({ }^{*} \mathrm{P}<0.05,{ }^{\# \#} \mathrm{P}<0.01\right)$ between TMZ group and $\mathrm{RA}+\mathrm{TMZ}$ group. $\mathrm{RA}$, retinoic acid; TMZ, temozolomide.

Our results suggested that the RA treatment enhanced the TMZ effects on inhibition of the U251 cell growth and arresting cell cycle progression at the G0/G1 phase. Besides, RA+TMZ treatment was able to significantly induce U251 cell apoptosis. Most importantly, the results of ultrastructural TEM analysis were consistent with autophagy protein levels, showing an extensive cytoplasm vacuolization and many single-membrane autophagolysosomes containing cellular debris. Western blot analysis and RT-PCR confirmed the downregualtion of Keap1/Nrf2/ARE signaling pathway involved in the mechanisms that the RA treatment could enhance the TMZ effects on $\mathrm{U} 251$ cells.
In spite of the advances in GBM therapies, the overall 5 year survival rate is still less than $5 \%$ (21). The efficacy of TMZ is often limited due to the resistance development (22). Studies have found that autophagy played a central role in the drug treatment of GBM. TMZ induces autophagy of glioma cells to produce anticancer effect $(23,24)$. It has been reported that autophagy has close connection with the chemoresistance in glioma cells (25-27). Even though autophagy had a suppressive effect on tumor in normal cells, the autophagy induction may facilitate tumor cells survival, resulting in the resistance to cellular death. However, autophagy could also be involved in the death mechanism that inhibited tumor progression based 
on the degree of cellular context and the DNA damage (22). Currently there is no consistent opinion on whether the autophagic death could be considered as a form of the programmed cellular death, or whether it could simply represent dying cells with the autophagic feature (28). In addition, the neurons and brain tissues were the major targets for illuminating how RA deficiency could influence the plasticity, differentiation, and neuronal neurogenesis (29-31), which may explained the reason for the altered behavior induced by the RA deficiency. However, the effects of RA deficiency on the glial cells remain elusive.

The new results in our present study were that the RA treatment in combination with TMZ in the human U251 cells could further inhibit the cells growth, arresting cell cycle progression at the G0/G1 phase, and significantly induced apoptosis of U251 cells. Moreover, after the RA+TMZ treatment of U251 cells, autophagy-associated proteins Beclin 1 and LC3B were significantly increased, and the ultrastructural TEM analysis were consistent with autophagy protein levels. Keap1/Nrf2/ARE expression was significantly downregulated, indicating the involvement in the mechanisms that RA treatment could enhance the TMZ effects on U251 cells. The close correlation between the U251 cells and the RA treatment in combination with TMZ administration may provide some experimental evidence for the possible effect of the RA+TMZ against the growth and proliferation of the U251 cells. The clear mechanisms underlying the RA+TMZ action and its utility for the treatment of GBM cancer in humans still need to be investigated further.

\section{References}

1. Hathout L, Ellingson BM, Cloughesy T and Pope WB: A novel bicompartmental mathematical model of glioblastoma multiforme. Int J Oncol 46: 825-832, 2015.

2. Huse JT and Holland EC: Targeting brain cancer: Advances in the molecular pathology of malignant glioma and medulloblastoma. Nat Rev Cancer 10: 319-331, 2010.

3. Bryukhovetskiy IS, Dyuizen IV, Shevchenko VE, Bryukhovetski AS, Mischenko PV, Milkina EV and Khotimchenko YS: Hematopoietic stem cells as a tool for the treatment of glioblastoma multiforme. Mol Med Rep 14: 4511-4520, 2016

4. Knisely JP and Baehring JM: A silver lining on the horizon for glioblastoma. Lancet Oncol 10: 434-435, 2009.

5. Nanegrungsunk D, Onchan W, Chattipakorn Nand Chattipakorn SC: Current evidence of temozolomide and bevacizumab in treatment of gliomas. Neurol Res 37: 167-183, 2015.

6. Messaoudi K, Clavreul A and Lagarce F: Toward an effective strategy in glioblastoma treatment. Part II: RNA interference as a promising way to sensitize glioblastomas to temozolomide. Drug Discov Today 20: 772-779, 2015.

7. Wait SD, Prabhu RS, Burri SH, Atkins TG and Asher AL: Polymeric drug delivery for the treatment of glioblastoma. Neuro-oncol 17 (Suppl 2): ii9-ii23, 2015.

8. Fu YS, Wang Q, Ma JX, Yang XH, Wu ML, Zhang KL, Kong QY, Chen XY, Sun Y, Chen NN, et al: CRABP-II methylation: A critical determinant of retinoic acid resistance of medulloblastoma cells. Mol Oncol 6: 48-61, 2012.

9. Sakoe Y, Sakoe K, Kirito K, Ozawa K and Komatsu N: FOXO3A as a key molecule for all-trans retinoic acid-induced granulocytic differentiation and apoptosis in acute promyelocytic leukemia. Blood 115: 3787-3795, 2010.

10. Manor D, Shmidt EN, Budhu A, Flesken-Nikitin A, Zgola M, Page R, Nikitin AY and Noy N: Mammary carcinoma suppression by cellular retinoic acid binding protein-II. Cancer Res 63: 4426-4433, 2003.
11. Niederreither K, Abu-Abed S, Schuhbaur B, Petkovich M, Chambon P and Dollé P: Genetic evidence that oxidative deriva tives of retinoic acid are not involved in retinoid signaling during mouse development. Nat Genet 31: 84-88, 2002.

12. Jacobs S, Lie DC, DeCicco KL, Shi Y, DeLuca LM, Gage FH and Evans RM: Retinoic acid is required early during adult neurogenesis in the dentate gyrus. Proc Natl Acad Sci USA 103: 3902-3907, 2006

13. Sui X, Chen R, Wang Z, Huang Z, Kong N, Zhang M, Han W, Lou F, Yang J, Zhang Q, et al: Autophagy and chemotherapy resistance: A promising therapeutic target for cancer treatment. Cell Death Dis 4: e838, 2013.

14. Paglin S, Hollister T, Delohery T, Hackett N, McMahill M, Sphicas E, Domingo D and Yahalom J: A novel response of cancer cells to radiation involves autophagy and formation of acidic vesicles. Cancer Res 61: 439-444, 2001.

15. Fulda $S$ and Kögel D: Cell death by autophagy: Emerging molecular mechanisms and implications for cancer therapy. Oncogene 34: 5105-5113, 2015.

16. Galluzzi L, Morselli E, Vicencio JM, Kepp O, Joza N, Tajeddine N and Kroemer G: Life, death and burial: Multifaceted impact of autophagy. Biochem Soc Trans 36: 786-790, 2008.

17. Codogno P and Meijer AJ: Autophagy and signaling: Their role in cell survival and cell death. Cell Death Differ 12 (Suppl 2): 1509-1518, 2005.

18. Zhang C, Jia X, Wang K, Bao J, Li P, Chen M, Wan JB, Su H, Mei Z and He C: Polyphyllin VII induces an autophagic cell death by activation of the JNK pathway and inhibition of PI3K/ AKT/mTOR pathway in HepG2 cells. PLoS One 11: e0147405, 2016.

19. Kabeya Y, Mizushima N, Ueno T, Yamamoto A, Kirisako T, Noda T, Kominami E, Ohsumi Y and Yoshimori T: LC3, a mammalian homologue of yeast Apg8p, is localized in autophagosome membranes after processing. EMBO J 19: 5720-5728, 2000.

20. Cao Y and Klionsky DJ: Physiological functions of Atg6/Beclin 1: A unique autophagy-related protein. Cell Res 17: 839-849, 2007.

21. Yan Y,Xu Z, Dai S, Qian L, Sun L and Gong Z: Targeting autophagy to sensitive glioma to temozolomide treatment. J Exp Clin Cancer Res 35: 23, 2016.

22. Zhang C, Zhu Q, He H, Jiang L, Qiang Q, Hu L, Hu G, Jiang Y, Ding X and Lu Y: RIZ1: A potential tumor suppressor in glioma. BMC Cancer 15: 990, 2015.

23. Zhang C, Cai Z, Liang Q, Wang Q, Lu Y, Hu L and Hu G: RLIP76 depletion enhances autophagic flux in U251 cells. Cell Mol Neurobiol 37: 555-562, 2016

24. Kanzawa T, Germano IM,Komata T, Ito H, Kondo Y and Kondo S: Role of autophagy in temozolomide-induced cytotoxicity for malignant glioma cells. Cell Death Differ 11: 448-457, 2004.

25. Shinojima N, Yokoyama T, Kondo Y and Kondo S: Roles of the Akt/mTOR/p70S6K and ERK1/2 signaling pathways in curcumin-induced autophagy. Autophagy 3: 635-637, 2007.

26. Natsumeda M, Maitani K, Liu Y, Miyahara H, Kaur H, Chu Q, Zhang H, Kahlert UD and Eberhart CG: Targeting notch signaling and autophagy increases cytotoxicity in glioblastoma neurospheres. Brain Pathol 26: 713-723, 2016.

27. Chen Y, Meng D, Wang H, Sun R, Wang D, Wang S, Fan J, Zhao Y, Wang J, Yang S, et al: VAMP8 facilitates cellular proliferation and temozolomide resistance in human glioma cells. Neuro Oncol 17: 407-418, 2015.

28. Pratt J and Annabi B: Induction of autophagy biomarker BNIP3 requires a JAK2/STAT3 and MT1-MMP signaling interplay in Concanavalin-A-activated U87 glioblastoma cells. Cell Signal 26: 917-924, 2014.

29. Bordin DL, Lima M, Lenz G, Saffi J, Meira LB, Mésange P, Soares DG, Larsen AK, Escargueil AE and Henriques JA: DNA alkylation damage and autophagy induction. Mutat Res 753: 91-99, 2013.

30. Wagner E, Luo T, Sakai Y, Parada LF and Dräger UC: Retinoic acid delineates the topography of neuronal plasticity in postnatal cerebral cortex. Eur J Neurosci 24: 329-340, 2006.

31. Baharvand H, Mehrjardi NZ, Hatami M, Kiani S, Rao M and Haghighi MM: Neural differentiation from human embryonic stem cells in a defined adherent culture condition. Int J Dev Biol 51: 371-378, 2007. 